\title{
Dos modelos explicativos de los procesos de composición escrita
}

\author{
Marlene Scardamalia y Carl Bereiter \\ Instituto de Estudios en Educación
}

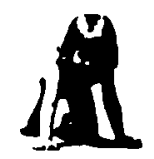

Resumen

\begin{abstract}
Se analizan dos modelos de los procesos de composición escrita Decir el conocimiento y Transformar el conocimiento con el propósito de captar las diferencias esenciales entre escritores expertos y novatos. La idea básica que subyace a estos modelos es que la principal diferencia entre los procesos de composición de expertos y novatos radica en la manera que el conocimiento es introducido en el proceso de composición y en lo que posteriomente le sucede a ese conocimiento. Se presentan además investigaciones educativas, cuya finalidad fue la de fomentar el desarmollo del modelo Transformar el conocimiento. Se supone que la tarea del cientifico cognitivista debe ser no sólo la de describir los procesos cognitivos sino la de encontrar aquellas diferencias que son significativas desde un punto de vista educacional. Si queremos ayudar a los alumnos a adquirir una estructura de «transformar el conocimiento» en los procesos de composición, es necesario llegar a modelos explícitos de competencia madura y poder diseñar procedimientos que transformen la competencia que los alumnos traen a la tarea espontánea. Los resultados de aplicar diversas estrategias educacionales sugieren que es posible transmitir los procedimientos complejos de solución de problemas que poseen los expertos, $y$ que al bacerlo se pueden ampliar las facultades de los alumnos para escribir. Sin embargo, los estudios educativos también indican que pasar de edecir el conocimiento» a «transformar el conocimiento» en la composición, no es un proceso de crecimiento sino de reconstrucción de una estructura cognitiva.
\end{abstract}

Palabras clave: Procesos de composición, Expertos y novatos, Estrategias instruccionales.

\section{Two explicative models for the processes of written composition}

\section{Abstract}

Two models of composing processes Knowledge telling and Knowledge transforming are set forth to capture the essential features that distinguish inmature composing from mature writers. The basic idea underlying the models is that the principal difference between mature and inmature composing is in bow knowledge is brought into the writing process and in what happens to knowledge in that process. The article deals also with instructional interventions which aim to belp the students move toward's a Knowledge transforming approsch. It is assumed that the applied cognitive scientist task is not merely to describe cognitive processes but to describe them in ways that capture educationally significant differences. The described instructional efforts are based on the premise that belping students move from a Knowledge telling to a Knowledge transforming approach to writing is an important and realistic educational objective. It will be argued that the importance of this objective extends beyond writing instruction, baving broad implications for the way students develop their knowledge.

Key words: Composing processes, Experts and novices, Intructional strategies.

Dirección de los autores: Instituto de Estudios de Educación. Ontario. Canadá. 
Una tarea central para los que trabajan en las ciencias cognitivas aplicadas es la de encontrar niveles de descripción que capten las diferencias entre los procesos lingüísticos que, desde el punto de vista educativo, son significativas. El campo de la investigación de los procesos de composición escrita ha surgido sólo recientemente. Y aunque los modelos sobre los procesos de composición se proponen proporcionar una descripción que se pueda aplicar a escritores de todos los niveles, la mayoría de los trabajos se llevó a cabo comparando entre escritores novatos y expertos (Burtis, Bereiter, Scardamalia y Tetroe, 1983; Flower y Hayes, 1980; Scardamalia y Paris, 1985). Esto ha creado una brecha entre la teoría y los datos. Los trabajos de investigación empiricos han generado una gran cantidad de datos sobre la diferencia entre expertos y novatos, pero los modelos asequibles han sido demasiado generales $y$, por lo tanto, de poca utilidad para ordenar esta masa de observaciones.

En este artículo expondremos dos modelos de procesos de composición que han sido desarrollados muy extensamente en trabajos anteriores (Scardamalia \& Bereiter, 1987). El primero modelo, intenta explicar los procesos de la composición «inmadura» y, el segundo modelo, aquellos de los escritores «maduros».

Utilizamos los términos maduro e inmaduro en lugar de expertos y novatos, porque uno de los grupos de referencia está formado por estudiantes próximos a la graduación o ya graduados y el otro por alumnos de la escuela primaria. Por lo tanto, muchos de los integrantes del grupo de referencia «maduro» serán, sin duda, expertos en el uso de la palabra escrita, y algunos integrantes del grupo «inmaduro» serán muy hábiles en la clase de composición que ejecuten.

Nuestro propósito es demostrar que la diferencia en el modo global de escribir de estos dos grupos es de un profundo significado educativo. Hemos denominado a estos dos modelos contrastados como «decir» $y$ «transformar» el conocimiento. Esta denominación refleja la idea de que la principal diferencia existente entre la composición madura e in- madura está en la manera de introducir el conocimiento y en lo que le sucede a ese conocimiento a lo largo del proceso de composición. Numerosos escritores experimentados aseguran que su comprensión de lo que están intentando escribir, crece y cambia durante el proceso de composición (Lowenthal, 1980; Murray, 1978; Odell, 1980). Estos efectos sobre la comprensión no son propiedades universales del proceso de composición, sino el resultado de ciertos procedimientos complejos de solución de problemas presentes en un modo de composición y ausentes en el otro.

Una forma posible de exponer estos modelos sería detallando las diferencias evolutivas entre expertos y novatos, mostrando de qué modo los dos modelos captan las diferencias en los procesos. Pero la cuestión no es tan simple ya que hay pocas diferencias inequívocas. Cuando se documenta el proceso de composición utilizando protocolos que registran el «pensamiento en voz alta» se comprueba que niños y escritores maduros proceden de forma diferente, particularmente en lo que respecta a la planificación de objetivos. Pero, sin una teoría que explique cómo es posible producir textos coherentes sin planificación, se podría desechar los descubrimientos - sostener, por ejemplo, que la carencia demostrada en el protocolo es un simple efecto del limitado lenguaje de los niños para expresar sus procesos mentales-. Por lo tanto, en lo que sigue hemos escogido la manera opuesta de exposición: explicaremos primero los modelos detalladamente y, luego, especificaremos las diferencias más evidentes. Posteriormente nos referiremos a la intervención educativa a la luz de estos modelos. Como ya se ha especificado, la tarea de las ciencias cognitivas aplicadas no es la de describir simplemente los procesos cognitivos, sino la de captar las diferencias educativas significativas. Los esfuerzos educativos que se describirán se basan en la premisa de que es necesario ayudar a los estudiantes a pasar de procesos de composición que consisten en «decir el conocimiento» a procesos de composición que consisten en «transformar el conocimientom. Este es un objetivo educativo 
práctico e importante porque va más allá de la enseñanza de la redacción y tiene claras implicaciones en la manera en que los alumnos desarrollan su conocimiento.

\section{PRIMER MODELO: «DECIR EL CONOCIMIENTO,}

Este modelo explica una manera de generar el contenido de un texto, a partir de un tópico sobre el que se ha de escribir y a partir de un género conocido (exposición de los hechos, opinión personal, instrucciones, etc.). El esquema del modelo en la figura 1 , muestra cómo la creación de un texto puede producirse bajo estas circunstancias, sin la necesidad de un plan u objetivo global para elaborar las restricciones del problema (Flower y Hayes, 1980), o sin los procedimientos de solución característico de los procesos maduros de composición (Hayes y Flower, 1980b).

FIGURA 1

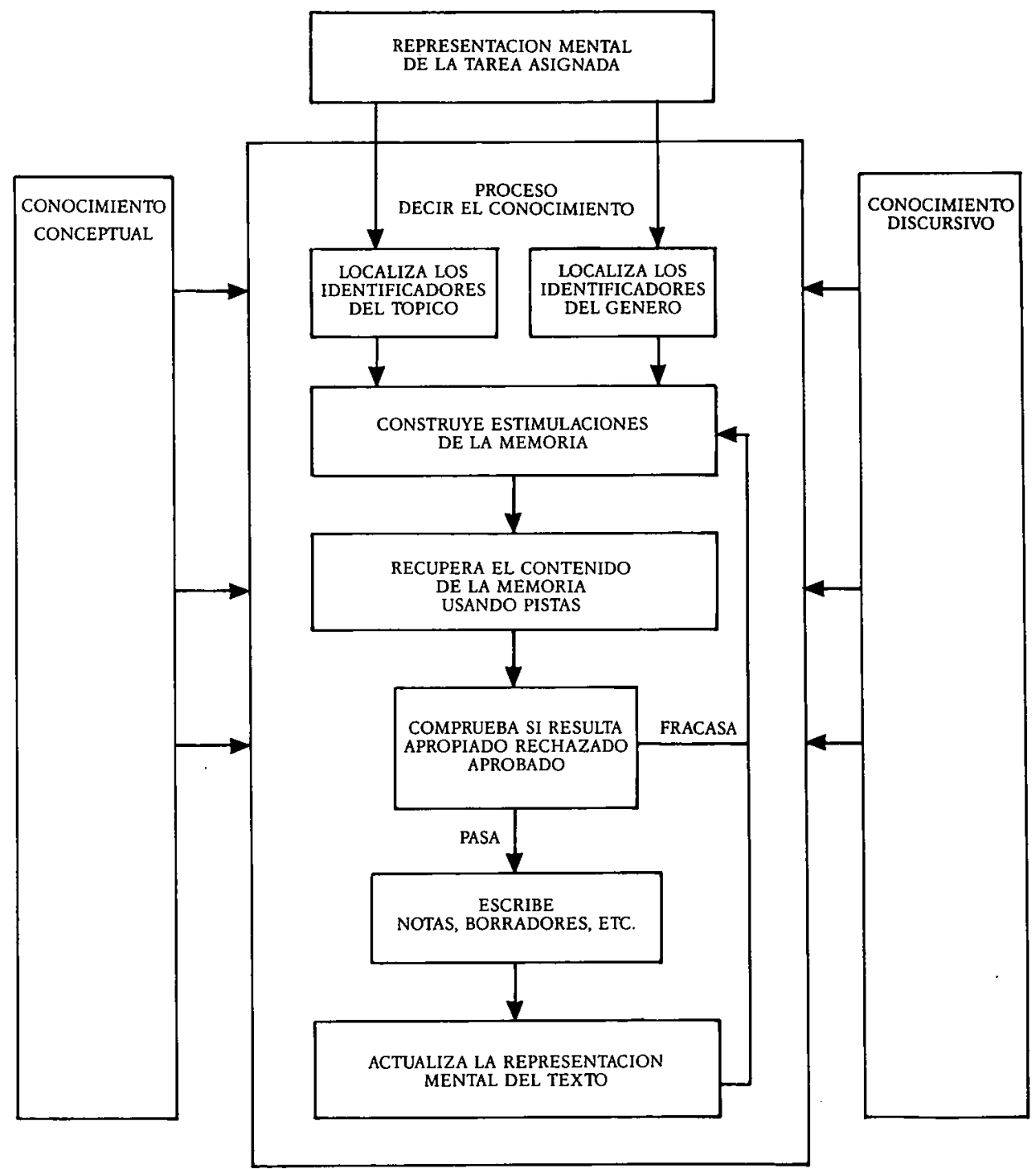

La estructura del modelo "decir el conocimiento». Este modelo es una adaptación del modelo, presentado con anterioridad, por Bereiter y Scardamalia (1985) 
El texto se genera de la siguiente manera: el escritor construye alguna representación de lo que se le ha pedido que escriba, luego, localiza los identificadores del tópico y del género. Por ejemplo, si se le ha solicitado que escriba sobre el siguiente tópico: «¿Deberían jugar chicos y chicas en el mismo equipo de deporte?", los identificadores del tópico podrían ser: equipo de deporte, igualdad y liberación femenina.

Estos idenficadores sirven como pistas para la búsqueda en la memoria y estas pistas ponen en marcha, automáticamente, conceptos asociados. Así, gracias a un proceso de activación propagadora, es posible utilizar en la composición la información que existe en la memoria (Anderson, 1983; Caccamise, en prensa; Collins y Loftus, 1975). Este proceso se inicia por medio de pistas deducidas de la propia tarea asignada. A pesar de que este proceso no garantiza que toda la información activada sea pertinente, hay una tendencia implícita hacia la pertinencia. Anderson lo explica de la siguiente manera: «La activación propagadora identifica y favorece la elaboración de la información más afín al contexto inmediato (o fuentes de la activación)» (Anderson, 1983, p.86). Por lo tanto, el texto producido por medio de este proceso exime al escritor de la necesidad de un control o un plan coherente.

Además de los identificadores tópicos, la tarea asignada también prescribe o implica el género. En el ejemplo sobre el equipo de deporte, el formato de la pregunta identifica el género requerido como ensayo de opinión. Según el modelo que estamos describiendo, los identificadores del género funcionan del mismo modo que los identificadores del tópico. Conducen a estimulaciones de la memoria, que operan en combinación con las estimulaciones de la memoria tópica, para aumentar la posibilidad de que la activación propagadora produzca el contenido apropiado. Por ejemplo, la estimulación-tópica combinada con la estimulación-género pueden ambas activar el contenido para que la primera frase del texto sea: «Creo que los chicos y las chicas deben jugar en el mismo equipo de deporte.»
Una vez que se ha producido algo de texto (ya sea mentalmente o por escrito) éste sirve como fuente adicional de identificadores de tópico y de género. Estos identificadores adicionales no sólo ayudan a la recuperación de contenidos sino que, aumentan la tendencia hacia la coherencia, ya que el próximo item que se recuperará se verá influenciado por los items previamente recuperados. En nuestro ejemplo, después de producida la primera afirmación, el conocimiento del joven escritor sobre el género «ensayo de opinión» producirá probablemente la estimulación de la memoria para dar la justificación. Esta estimulación, junto a las estimulaciones asociadas con chicas, chicos, deportes e igualdad, podrían añadirle al texto: «Las chicas son tan capaces para el deporte como los chicos». Este proceso: pensar-decir, continúa hasta que se acabe la hoja o hasta que se agoten las ideas que vienen a la memoria.

Se advertirá que en el modelo «decir» el conocimiento, la coherencia y la buena forma no dependen de la aplicación deliberada o consciente del conocimiento sobre el mundo o del conocimiento sobre los géneros literarios. Sino que éstas resultarían de procesos automáticos puestos en marcha gracias a esta actividad.

\section{SEGUNDO MODELO: ARANSFORMAR EL CONOCIMIENTO»}

En la figura 2 se podrá observar un diagrama del proceso de composición madura según el modelo «transformar el conocimiento».

Este modelo no es una mera elaboración del modelo anterior, aunque tampoco está completamente desligado de él. Más bien, el nuevo modelo contiene al anterior como un subproceso, introduciéndolo dentro de un proceso complejo de solución de problemas.

Este proceso de solución de problemas implica dos clases distintas de «espaciosproblema» (Scardamalia, Bereiter, Steinbach, 1984). Concebimos un espacio de problema, de acuerdo con Newell (1980), como una entidad abstracta formada por un número de es- 
FIGURA 2

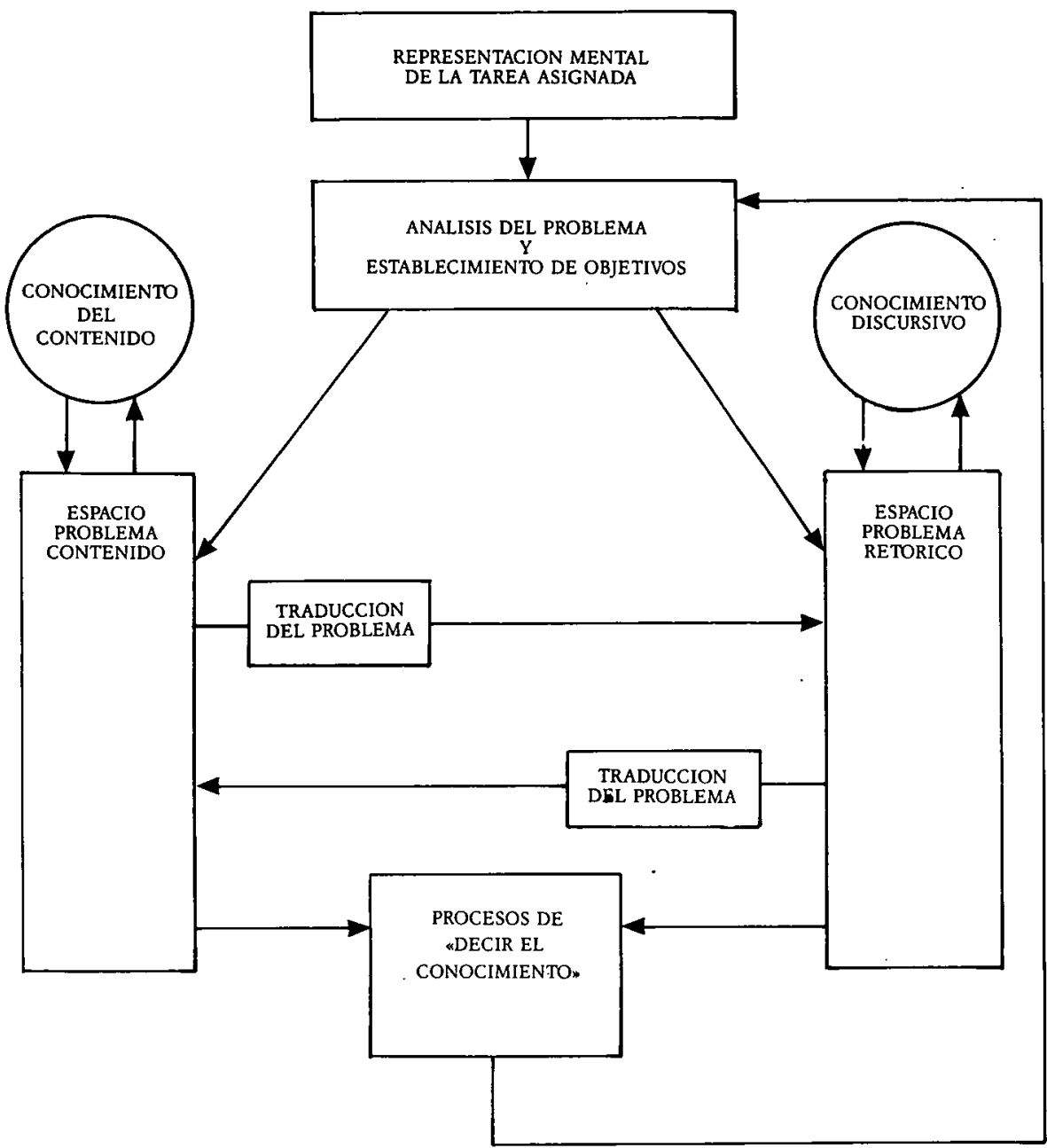

La estructura del modelo transformar el conocimiento

tados de conocimiento y de operaciones, siendo el efecto de las operaciones el de producir movimiento a través de este espacio, desde un estado de conocimiento hacia otro. Estos dos espacios son: de contenido y retórico. En el de contenido, los estados de conocimiento se pueden caracterizar como creencias y las operaciones como las deducciones o las hipótesis que conducen de un estado de creencia a otro. En el espacio retórico, los estados del conocimiento son las múltiples representaciones que incluyen al texto y sus objetivos subordinados; y las operaciones son aquellas que alteran el texto, los objetivos o las relaciones entre el texto y los objetivos.

Tal distinción entre ambos espaciosproblema queda implícita en las concepciones clásicas de la retórica. La retórica, según Bryant (1965, p.18), es «distinta del saber que utiliza». No se ocupa del contenido conceptual del discurso per se, sino, más bien, de las «relaciones entre el contenido conceptual y los pensamientos, los sentimientos, los motivos y el comportamiento humano». En el mo- 
delo «transformar el conocimiento», este «saber» opera dentro del espacio del contenido. El espacio retórico por lo tanto se ocupa de cumplir los objetivos discursivos así como de las relaciones entre el contenido y las posibles reacciones del lector.

La transformación del conocimiento se produce dentro del espacio del contenido pero, para que ésta se realice debe haber una interacción entre el espacio del contenido y el espacio retórico. La clave consiste en traducir problemas del espacio retórico en subobjetivos a conseguir dentro del espacio del contenido y viceversa. Así, las dificultades que se pueden encontrar dentro del espacio retórico por ejemplo, para que una afirmación sea convincente, podrían traducirse en subobjetivos que produzcan entre otros: la explicitación de los motivos de una creencia, la ejemplificación de los conceptos, el desarrollo de los pasos intermedios en la cadena del razonamiento, los argumentos en contra de otra creencia, etc. Las operaciones llevadas a cabo dentro del espacio del contenido para conseguir tales subobjetivos podrían, a su vez, producir cambios en: la creencia del escritor o en sus elaboraciones; podrían provocar nuevas conexiones o modificaciones en sus objetivos hacia estudios futuros. De este modo, la interacción dialéctica entre los dos espacios-problema podría producir cambios en el contenido y en la organización del conocimiento del escritor.

Una pregunta que el modelo procura contestar es: ¿cómo ocurre la generación de ideas que corrientemente aparecen en los protocolos como un suceso espontáneo? El modelo sostiene que las necesidades retóricas se transforman en sub-objetivos relacionados con el contenido. Suponemos que no se pueden buscar en la memoria ideas etiquetadas por «que no ocupe toda una página» o «que no parezca un estereotipo de los profesores». Criterios de esta clase deben ser traducidos en subobjetivos según el modo en que la información se codifica en la memoria. Por lo que el sub-objetivo podía ser: «encontrar un ejemplo de la idea $\mathrm{X}$ que sea concreto, que no sea complejo y que no se asocie mayormente con Z». Tal sub-objetivo podría entonces proveer estimulaciones de la memoria que despertarían, probablemente, contenidos que satisfagan las especificaciones originales.

Suponemos que la recuperación de la información en la memoria, es igual a la implicada en el proceso «decir el conocimiento» - concierne a la activación propagadora desde nodos activados por la estimulación de la memoria, pero hay muchas más estimulaciones de la memoria que reflejan sub-objetivos retóricos- L La consecuencia es que la información recuperada no sólo debe adaptarse a las necesidades del tópico y del género, sino que debe tener buenas probabilidades de adaptarse a las restricciones específicas originadas por el análisis del escritor del problema retórico (Flower y Hayes, 1980). Más aún, al encontrarse con estas restricciones distintivas, la información recuperada probablemente llamará la atención sobre algunas relaciones nuevas. Este nuevo item del conocimiento puede traducirse a su vez, en un nuevo sub-objetivo para la composición - por ejemplo, el objetivo de modificar el plan para incorporar en el texto una discusión de la nueva relación-. La prosecución de este nuevo sub-objetivo puede acarrear nuevos problemas retóricos, que se traducen, a su vez, en nuevos sub-objetivos en el espacio del contenido, y así sucesivamente. Esto no sucede en el proceso «decir el conocimiento», porque, en realidad, no se trata de solucionar problemas retóricos. El contenido recuperado de la memoria pasa directamente a formar parte de la composición, o bien es rechazado y, entonces, se recupera otro contenido. De todos modos, en ninguno de estos dos sucesos existe un proceso dialéctico que lleve a la elaboración de sub-objetivos para la composición, o que conduzca una búsqueda en nuevas áreas de la memoria.

\section{LOS DOS MODELOS DE COMPOSICION DESDE LA PERSPECTIVA PSICOLOGICA}

Hemos sostenido que estos modelos representan lo que en realidad ocurre en las mentes de los típicos escritores inmaduros y ma- 
duros. Es ahora el momento de tratar más explícitamente esta cuestión. Aunque algunos autores (por ejemplo Chomsky, 1980) han recalcado que una teoría cognitiva puede ser válida si explica un conjunto de hechos, aun no guardando ninguna semejanza con lo que en realidad ocurre en la mente. Sin embargo, para que los modelos cognitivos puedan llegar a ser prometedores como guías de la enseñanza, deberían cumplir también esta última condición. O sea que, para corresponder con cualquier concepción razonable del crecimiento educativo, los modelos cognitivos deben poseer realidad psicológica. No obstante, alegar la realidad psicológica de un modelo es muy rotundo y no puede hacerse en las primeras fases de verificación de un modelo cognitivo. La plausibilidad psicológica es un alegato mucho más inmediatamente accesible de un modelo para describir lo que en realidad ocurre en la mente. Preguntas sobre la plausibilidad psicológica se pueden formular con respecto a los modelos «decir el conocimiento» y «transformar el conocimiento»: ¿Son los modelos compatibles con la experiencia subjetiva de escribir de la gente? y ¿existe alguna información plausible de cómo las estructuras cognitivas descritas por los modelos pueden llegar a ser adquiridas?

\section{Los que escriben informan sobre el proceso de composición}

Los informes que hemos obtenido sobre los procesos de composición de los niños concuerdan con el modelo «decir el conocimiento». Para ejemplificar exponemos aquí la estrategia de composición de un alumno de sexto grado:

«Tengo un montón de ideas y escribo hasta que mi suministro de ideas se agota. Entonces intentaría pensar en más ideas hasta el punto en que no puedes obtener ninguna idea más que merezca la pena llevar al papel y entonces lo acabaría».

Un informe similar de otro alumno de sexto grado:

«Tengo un montón de buenas ideas y empiezo a escribir las más importantes primero, porque son las que tengo al frente de $\mathrm{mi}$ mente. Entonces las menos importantes em- piezan a aparecer a las tres cuartas partes de la página y quizás un montón de esas... un enjambre de esas... así que podría escribir tantas como me sea posible. No lleva tanto tiempos.

A primera vista parecería que este alumno percibe la importancia relativa que existe entre las ideas, lo que podría implicar juicios retóricos sofisticados. Pero, de hecho, la creencia de que las ideas más importantes son las primeras que se nos vienen en mente, es completamente congruente con un proceso de activación propagadora que empieza con la información más próxima a las sugerencias relativas al contexto.

En contraposición con este proceso de generación directa descrito por estos escritores novatos, se encuentra el proceso más recursivo y lleno de problemas descrito por algunos escritores expertos. Exponemos, como ejemplo, la descripción de Aldous Huxley del proceso de construcción de una pieza de ficción:

«Generalmente, escribo todo muchas veces. Todos mis pensamientos son segundos pensamientos. Y corrijo bastante cada página, o la reescribo muchas veces sobre la marcha... Las cosas se me aparecen en trozos, y cuando los trozos aparecen tengo que trabajar duro para hacer de ellos algo coherente. (Cita de Writers at Works, Escritores trabajando, segunda serie, 1963, p.197).

No todos los escritores expertos trabajan sobre sus textos del mismo modo que Huxley. Algunos cuentan que primero producen borradores casi del mismo modo como lo hacen los alumnos de sexto grado, pero luego los corrigen exhaustivamente. Otros, elaboran sus ideas por medio de conferencias y discusiones, y así, cuando finalmente ponen sus ideas por escrito, un proceso directo de decir el conocimiento es suficiente. Estas parecen ser variaciones de transformar el conocimiento más que indicios de un proceso radicalmente diferente.

Los escritores expertos atestiguan, con frecuencia, sobre el papel que juega el proceso de composición en el desarrollo de sus pensamientos y de su conocimiento. C. Day Lewis (citado en Murray, 1978, p. 102) llega a sostener que «no escribimos para ser enten- 
didos, escribimos para entender». Algunas veces los escritores se refieren al escribir como a un proceso de descubrimiento, o expresan la sensación de que una pieza de escritura adquiere autonomía hasta tal punto que influye sobre el pensamiento del escritor, en lugar de que la influencia suceda sólo en la dirección contraria.

Dentro del modelo «trasnformar el conocimiento» tales impresiones pueden ser atribuidas al efecto que tienen los problemas generados en el espacio retórico sobre la búsqueda en el espacio del contenido.

Nuestros informadores juveniles no nos hablan de los efectos liberadores o generadores de descubrimientos del escribir.

Los escritores jóvenes tienden, más bien, a describir la escritura como un proceso de decir el conocimiento, en el cual los únicos problemas son los problemas de expresar lo que ya está formado en sus mentes. Un ejemplo de la visión de un alumno de sexto grado:

«Mi principal idea... es hacer que mis ideas queden claras a otro, pero sólo a otro y no a mí... Para mí, es automáticamente claro especialmente si me pongo en el lugar del otro... porque yo lo escribìn.

La mayoría de los escritores jóvenes no son ni tan lúcidos ni tan explícitos como los citados aquí. Estos jóvenes escritores fueron, de hecho, seleccionados para estas entrevistas porque eran escritores particularmente talentosos. Por lo que es aún más extraordinario que al describir el proceso de composición de forma global, lo hagan en términos que son mucho más compatibles con el modelo «decir el conocimiento» que con el de «transformar el conocimiento».

\section{¿Cómo llegan los modelos a la mente?}

Los seres humanos, incluyendo los niños, son dignos de atención por su predisposición a la búsqueda de objetivos y a la solución de problemas. Debemos aclarar que el modelo «decir el conocimiento» no sostiene que los jóvenes no tengan objetivos o intereses al escribir. Sostiene, simplemente, que su sistema de ejecución carece de recursos para introducir, de un modo activo, estos objetivos o in- tereses en el proceso de composición. De todos modos, parece poco plausible que los niños puedan desarrollar tal acercamiento a la composición.

El gran problema con el que se encuentran los escritores novatos es el de convertir el sistema de producción del lenguaje que han desarrollado para la conversación - un sistema que depende en todos sus niveles de inputs del medio ambiente social - en un sistema capaz de funcionar autónomamente. Esta transformación implica, encontrar caminos para sostener el discurso más allá del patrón alternante de conversación, caminos para activar y buscar en los almacenes de la memoria, en ausencia de las contribuciones de otras personas al discurso, y desarrollar esquemas que garanticen su adecuación, coherencia e integridad en ausencia de una audiencia que responda.

Cuando los niños abordan este problema durante los primeros años escolares, tienen desventajas debidas a su limitado conocimiento $\mathrm{y}$ al hecho de que sus habilidades con la escritura, la propia caligrafía y la ortografía, no son todavía automáticas $\mathrm{y}$, por lo tanto, hacen que su esfuerzo mental se aparte de aquellos aspectos de la producción de un texto que poseen un nivel más alto. En consecuencia, cualquier solución al problema de generar un discurso escrito, será la que implique menos conocimiento y la que economice esfuerzos mentales. Economizar esfuerzos mentales significa: ocuparse de la menor cantidad de aspectos de la tarea, reducir los procedimientos a rutinas, evitar los procedimientos recursivos en favor de los lineares que permiten procesar los datos una vez y luego ignorarlos, y evitar el análisis en función de medio y fines.

Para todos estos puntos, el modelo «decir el conocimiento» representa una estrategia exitosa. Todo lo que requiere es un conjunto de esquemas que especifiquen los elementos estructurales apropiados para los textos y algunos nodos en la memoria capaces de ser activados por las pistas del tópico asignado. Sus exigencias a la capacidad de procesamiento son mínimas. El resultado es un procedimiento altamente eficiente, capaz de iniciar 
la composición pocos segundos después de que se haya asignado un tópico y de sostener el discurso dentro de los límites temáticos y estructurales.

Pensamos que el modelo «decir el conocimiento» es, por lo menos, un resultado plausible de la situación en la que se encuentran los niños en los primeros años de alfabetización. Lo más difícil de explicar en términos de plausibilidad es el surgimiento de una estructura cognitiva que se asemeje al modelo «transformar el conocimiento». Debe quedar claro que, en las figuras 1 y 2 , el modelo «transformar el conocimiento» no es una consecuencia del modelo más primitivo. Más bien es una estructura nueva, que incluye al modelo más primitivo como componente.

Una posibilidad es postular una diferencia en las personas desde el principio; las personas que llegan a escribir según el segundo modelo no pasarían necesariamente. Tanto en los casos sobre escritores precoces (Britton, 1982; Calkins, 1979, 1983), como los informes retrospectivos de escritores adultos expertos (Scardamalia y Bereiter, 1982), proveen fundamentos a estas especulaciones. En secciones posteriores de este artículo describimos los esfuerzos educativos que se han hecho para llevar a los estudiantes desde el modo de escribir «decir el conocimiento» al modo «transformar el conocimiento». A pesar de que los resultados de estos esfuerzos sustentan la creencia de que las estrategias para transformar el conocimiento pueden ser aprendidas, dejan sin contestar la pregunta de cuán lejos puede llegar la educación en su intento. Este es, a nuestro juicio, el desafío primordial con el que se enfrentan las investigaciones del cognitivismo aplicado a los procesos de composición.

\section{LAS CARACTERISTICAS DE LA COMPOSICION ESCRITA EN EXPERTOS Y NOVATOS}

En esta sección trataremos tanto los defectos que se observan en los escritores inmaduros (falta de adaptación a la audiencia, de planificación y de revisión), cuanto las carac- terísticas positivas, ya que ambos requieren una explicación.

\section{Acerca de los textos}

Ciertas características del texto son afectadas por el modo de producción:

1. Coherencia tópica. Los textos generados por el modelo «decir el conocimiento» tenderán a ceñirse a sus tópicos más simples. McCutchen y Perfetti (1982) encontraron, en textos escritos por alumnos de cuarto grado, que las frases eran coherentes con el tópico simple pero que faltaban otros encadenamientos entre las ideas - resultado compatible con la premisa de que el contenido es recuperado de la memoria, en primer lugar, por medio de pistas tópicas y estructurales-. El típico resultado de esta coherencia tan limitada es que un texto sobre si la televisión es una buena o mala influencia para los niños, tratará sobre la televisión y los niños, pero no necesariamente sobre la influencia de ésta sobre aquellos, lo que sería un encadenamiento de ideas mucho más complejo.

2. Buena forma. El modelo «decir el conocimiento" predice que los textos tenderán a ajustarse a los requisitos estructurales de los géneros literarios, aunque no alcancen, necesariamente, sus objetivos. Por lo tanto, en la escritura persuasiva, producirá, mínimamente, una afirmación de creencia acompañada de una lista de justificaciones, pero no una línea argumentativa desarrollada. En las evaluaciones de la composición persuasiva (National Assessment of Educational Progress, $1980 \mathrm{a}, 1980 \mathrm{~b})$ se ha visto que los textos que cuadran con esta descripción son el típico modelo tanto para los jóvenes de 13 años como para los de 17 años.

3. Prosa basada en el escritor. La escritura típicamente principiante fue diagnosticada por Flower (1979) como «prosa basada en el escritor" - una prosa que refleja el curso del pensamiento del escritor en vez de adaptarse al curso del pensamiento del lector-. Flower observó que esta clase de escritura es apropiada para un primer borrador, pero que con los escritores principiantes tiende a persistir en la propuesta final. Podemos predecir tal 
resultado del modelo «decir el conocimiemto", porque el proceso decir el conocimiento no provee medios como para que el escritor pueda, deliberadamente, aplicar su conocimiento acerca del lector. Así, los esfuerzos dicen que para adaptar su escritura a las características del lector, deberán depender del dominio de la estructura superficial (ej. el control de registro, traspasado de las habilidades del lenguaje oral) pero no comprenderán la habilidad de reorganizar la información de acuerdo con las necesidades del lector.

\section{Acerca del tiempo necesario para comenzar a escribir}

Según el modelo «decir el conocimiento» no se establecen objetivos o planificación alguna, por lo tanto, el tiempo para empezar deberá ser el tiempo que lleva recuperar un primer dato cuyo contenido cuadre con los requisitos del tópico y del género. El corolario es que el tiempo de puesta en marcha no debe variar con otros requisitos.

Según un trabajo de Zbrodoff (1984, citado en Scardamalia y Bereiter, 1987) los alumnos de quinto grado se comportan exactamente como es de esperar del modelo «decir el conocimiento». Los tiempos de puesta en marcha son muy cortos, sólo unos pocos segundos y no varían ni con el tiempo permitido ni con la longitud del texto que debe ser generado. Por el contrario, los adultos muestran adaptaciones consecuentes con una aproximación a la composición mucho mejor planificada. Cuanto más tiempo se les permite, más tiempo emplean antes de comenzar a escribir, y cuando no se impone ninguna coerción de tiempo, emplean incluso mucho más tiempo que bajo los límites más liberales de tiempo. Sus tiempos de puesta en marcha aumentan, también, de acuerdo con la longitud de la historia que se les ha pedido.

\section{Acerca de la confección de borradores}

En un estudio de Burtis y otros (1983), se les pidió a los alumnos que planifiquen y anoten antes de escribir lo que se les había asignado. Como el modelo «transformar el conocimiento», se utiliza para generar texto, es fac- tible que las notas de los alumnos inmaduros sean muy parecidas al propio texto. Se espera, sin embargo, que los alumnos que siguen el modelo «transformar el conocimiento", expusieran en sus notas una variedad de ideas que luego no incluirán en el texto.

Los borradores resultantes son, esencialmente, textos escritos en forma de lista. En contraposición, las notas de los alumnos graduados se parecen más a «hojas de cálculo». Los escritores captan las ideas a diversos niveles de abstracción, las evalúan y construyen estructuras con ellas.

\section{Acerca de la planificación}

Lo que el joven escritor inmaduro tiene en mente mientras escribe debe ser muy parecido a lo que aparece en la hoja. Sin embargo, el modelo «transformar el conocimiento» sugiere que en los escritores maduros la actividad es mayor; esta actividad se pone de manifiesto en los protocolos registrados al pensar en voz alta y no está directamente expuesta en el texto. Un trabajo realizado analizando protocolos de planificación en voz alta da inicios cuantitativos de las diferencias adulto-niño. (Figura 3).

Las barras oscuras muestran el número medio de palabras según protocolos producidos durante la planificación de las composiciones. Las barras blancas muestran el número medio de palabras en los ensayos correspondientes. En todas las edades, los escritores dicen más de lo que escriben, pero la diferencia en los adultos es, proporcionalmente, mucho mayor.

\section{Acerca de la revisión}

Otra manifestación de los procesos mentales de composición son los cambios que hacen los escritores en sus textos. Un manuscrito típico de la escuela primaria, no mostraría revisión alguna (National Assessment of Educational Progress, 1977), y existe una tendencia en todos los niveles escolares a que las revisiones sean meramente «cosméticas» (Nold, 1981). Sin embargo, con apoyo, los alumnos de la escuela primaria empiezan a hacer revisiones de cierta importancia (Berei- 
FIgURA 3

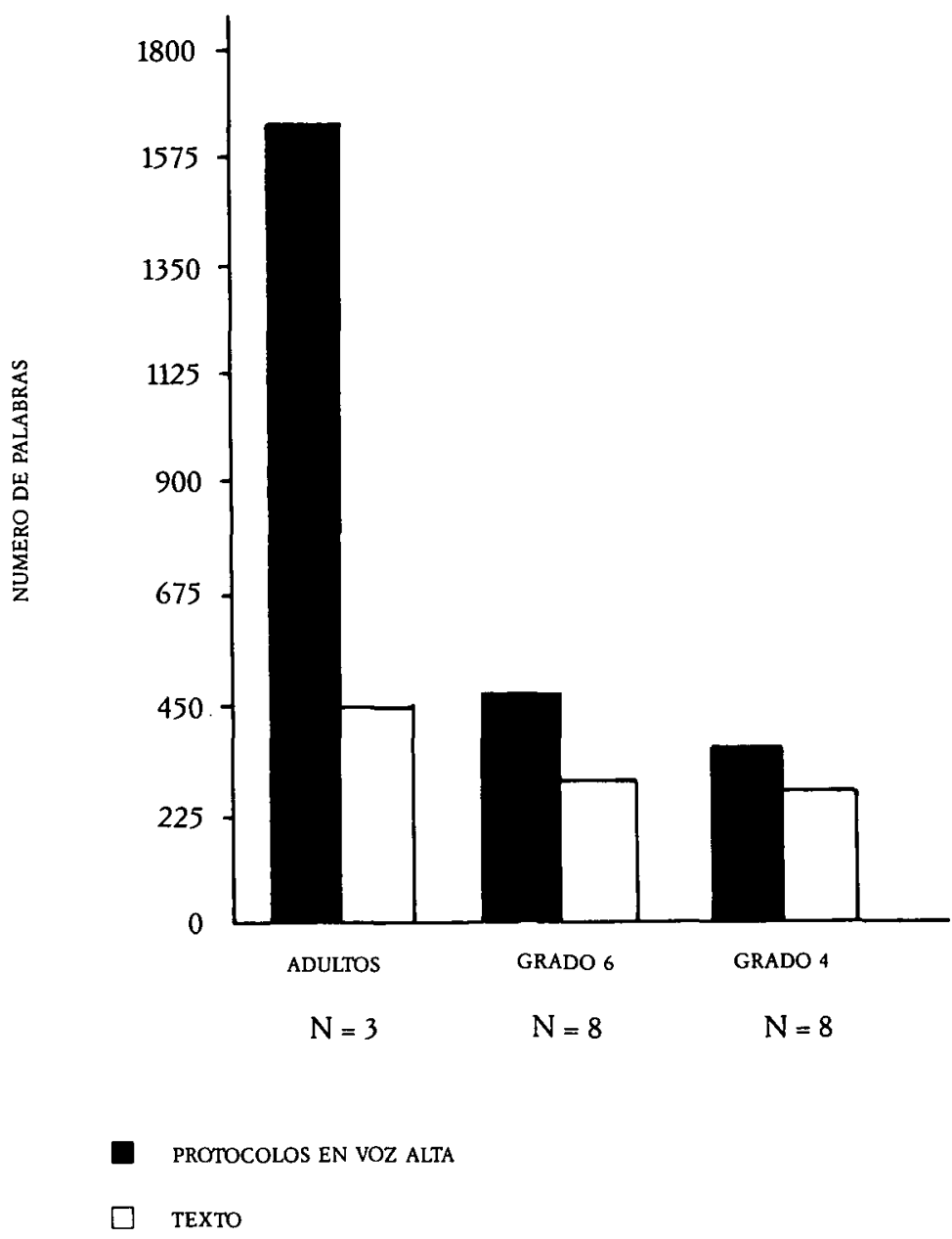

Número medio de palabras según protocolos producidos planificando sus composiciones comparado con los promedios de palabra en los textos resultantes

ter y Scardamalia, 1987, cap. 11; Calkins, 1983; Graves, 1979). Durante seis semanas de instrucción grupal fue posible aumentar el número de cambios conceptuales que los alumnos hacían en sus textos; estos se vieron reflejados en progresos significativos para todos menos dos de los alumnos, comparados los textos antes y después de la intervención.
Los cambios consistieron en: agregar una frase introductoria, agregar una conclusión, proveer información descriptiva adicional o introducir información que faltaba.

La figura 4 nos muestra el texto original y las revisiones hechas por un alumno de sexto grado, durante este experimento educacional de seis semanas. 
FIGURA 4

Knowledge telling and transforming in writing

DO YOU THINK THAT CHILDREN SHOULD BE ABLE TO WATCH AS MUCH TELEVISION AS THEY WOOLD LIKE?

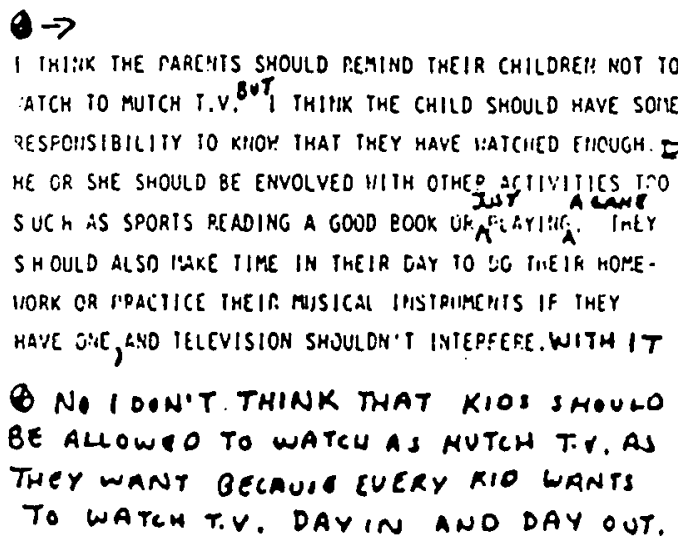

El texto original y las revisiones de un alumno de sexto grado que ba participado durante seis semanas en un programa de instrucción

Además del mejoramiento del estilo, el alumno muestra un adelanto estructural importante, al añadir una frase tópica que une el resto del texto. Sin embargo, con respecto a la distinción entre «decir el conocimiento» y «transformar el conocimiento», lo que debemos notar es que incluso estos progresos, representan modos alternativos de decir lo mismo $o$ añadiduras, más que transformaciones de la información. Por lo tanto, las revisiones no indican ninguna superación real del modelo «decir el conocimiento». La clase de reprocesamiento que no parece progresar en los escritores inmaduros, es aquella que implica objetivos e ideas principales (Perl, 1979; Sommers, 1980).

El tema de la revisión muestra lo valioso que es tener un modelo coherente de la composición inmadura. Los maestros que enseñan a escribir han tendido a ver la revisión como una parte importante aunque diferente del propio proceso de producción que los alumnos, por alguna razón, evitan. Sin em. bargo, los esfuerzos directos para conseguir que los alumnos revisen sus textos, han tendido a ser ineficaces (Faigley y Witte, 1981). Revisiones significativas presuponen un sistema de objetivos y procedimientos de búsqueda de objetivos. Los alumnos que no po- seen tal sistema necesitan algo más que el estímulo para revisar y necesitan también pautas para mejorar sus textos. Es decir, necesitan ayuda para desarrollar un sistema en el que la revisión pueda ser un proceso dirigido hacia un objetivo. La planificación y la adaptación a una audiencia, más allá de un nivel superficial, también dependen de un sistema así. Pero, para los alumnos que no poseen tal sistema, las lecciones sobre cómo esbozar y tomar notas o el ejercitarse en escribir para audiencias diferentes, es probable que no ayuden a superar la verdadera dificultad.

\section{LAS REPRESENTACIONES MENTALES CORRESPONDIENTES A CADA MODELO}

En los escritores inmaduros, el texto escrito parecería reflejar completamente al texto representado en la mente, mientras que, en los escritores maduros, el texto escrito correspondería sólo a una parte de las representaciones mentales. La otra parte incluiría codificaciones más abstractas del contenido del texto, de estructuras, objetivos, estrategias, etc. El tema sobre la clase de representaciones 
mentales de que disponen los escritores y lo que son capaces de hacer con ellas, es fundamental para entender, psicológicamente, la capacidad de escribir (Flower y Hayes, 1984). Modelos como los que se exponen en las figuras 1 y 2 , proveen un marco para la investigación de este tema, pero no aportan respuestas. Un marco teórico, sin embargo, es necesario porque las representaciones mentales no pueden ser observadas sino que deben ser deducidas de evidencias indirectas. En esta sección, tratamos dos clases de evidencias indirectas, que pueden ser interpretadas dentro del marco de los modelos «decir el conocimiento" y «transformar el conocimiento».

\section{Cómo se recuerda un texto}

La utilización de protocolos de recuerdo (una relación detallada de los procedimientos se encuentra en Scardamalia y Paris, 1985) es un medio indirecto para probar las hipótesis sobre las representaciones mentales de un texto. La suposición es que, al tratar de recordar cosas sobre sus textos, los escritores utilizan cualquier conocimiento almacenado que esté conectado con lo que ellos están tratando de recordar. Por esta razón, suponemos que los escritores maduros utilizan caminos complejos para recuperar la información textual, pasando por representaciones de toda clase, mientras que los escritores inmaduros utilizarían una menor variedad de representaciones y dependerían más del recuerdo directo de lo que se les haya pedido.

\section{El recuerdo del contenido}

A doce escritores de tres edades diferentes se les solicitó que piensen en voz alta mientras intentan recordar las palabras exactas de un texto que habían escrito anteriormente. Una idea aproximada de la cantidad de información utilizada desde fuera del texto, puede ser obtenida al comparar el número de palabras en el protocolo registrado al pensar en voz alta con el número de palabras en los textos. Estos datos se exponen en la figura 5 .

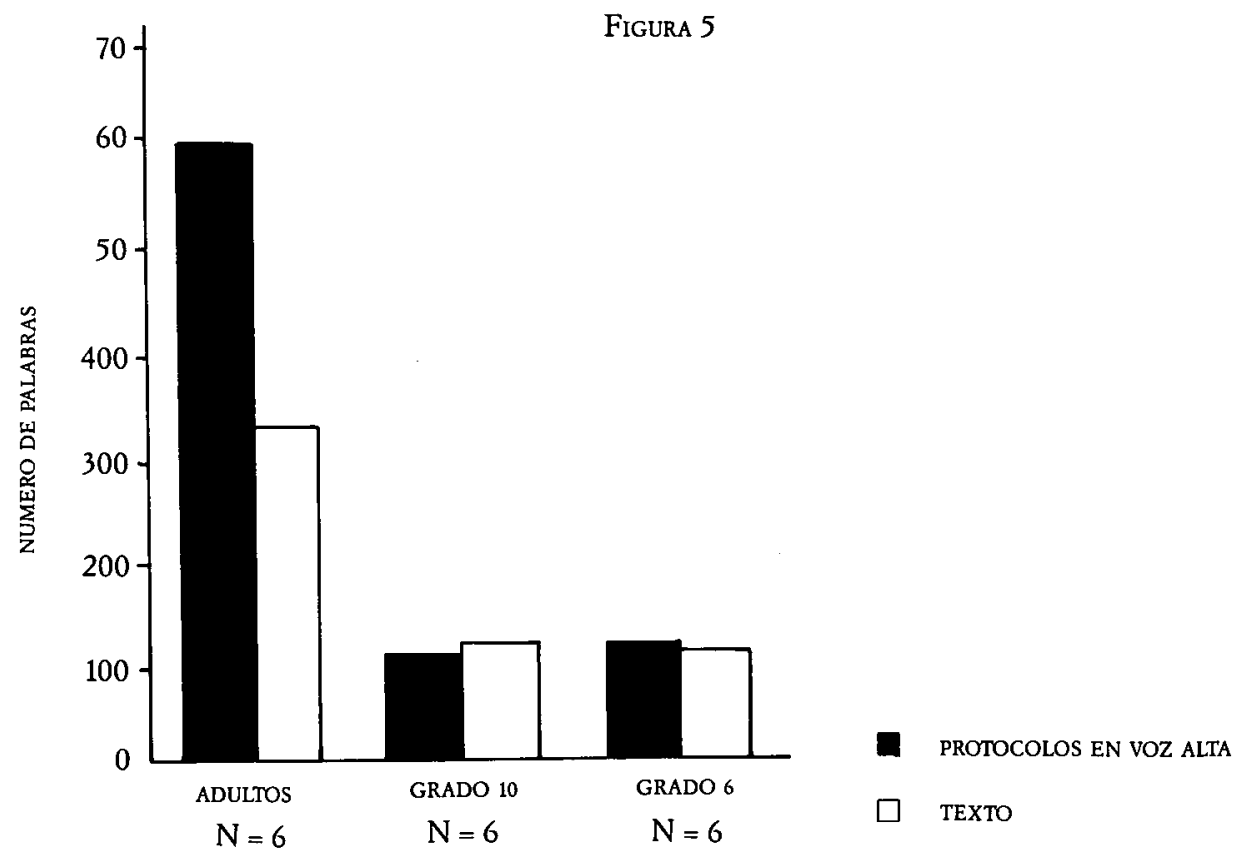

Número medio de palabras de los protocolos en voz alta de sujetos verbalizando el recuerdo de textos escritos comparado con el promedio de palabras en textos reales 
Los grupos son de adultos, alumnos de décimo grado y de sexto grado ( $\mathrm{n}=6$ por grupo). La otra mitad de los participantes recibió un tratamiento experimental que pudo haber influido en su rendimiento (detalles del estudio completo se encuentran en Scardamalia y Paris, 1985). Sólo los adultos utilizaron, como promedio, más palabras, para recupe- rar lo que estaba escrito en el texto, de las que utilizaron para escribirlo.

Podemos identificar las causas de las diferencias en el funcionamiento de los adultos y de los niños, si tenemos en cuenta la clase de actividad que ocurre en los protocolos de recuerdo. En la figura 6 aparece el protocolo de un adulto.

\section{FIGURA 6}

Topie: Should Children be Allowed to Choose What Subjects They Study in School

... I canitremember what the next sentence was, out the kenteral idea uas as a result of all of this the school could no lonker perform the function that it was performing in the past, and therefore was loft in a semi-chaotic situation. I did not in fast include that idea. What l just said nuw was not in there. It has not been bruught out. come to think of it. So then 1 said sumething like a lot of these changes are reflected in the demise of a core curriculum, and a reluciance to use, and that "asn't the wurd. but somehow standardized testing to determine achievement was no longer existent. And I don't quile remember whal I said. From there I think I went nn (1) a third paragraph where I was. trying codraw those things logether to say something like although it can be argued that a child's desirey, and something or other, should snmething lo the elrect that although it can be argued that the child should be able to choose his own subjects because this satisfies his needs and desires, it can also be arkued that by doing so .. 1 can't remember what 1 sald. I can't remember the argunient of the other side. I was struggling with it, and it wasnit all that clear. Then the rest ui il is all a jumble. That's where! was running into further problems. the poinc that I was trying to make, was ...

Parte de un protocolo de un adulto mientras recuerda su texto

El material en negrita reproduce o parafrasea el material que finalmente fue llevado al ensayo. El resto señala la solución de problemas: objetivos y claves se reconstruyen y se utilizan como ayuda para recuperar lo que se había dicho en el texto. El siguiente extracto muestra el uso de objetivos, de claves y de la representación estructural, para poder re- cordar: «De ahí creo que seguí hasta un tercer párrafo, donde estaba intentando poner juntas todas esas cosas, decir algo así como...» (sigue una declaración de la clave de la próxima parte). En contraposición, la figura 7 muestra el protocolo de recuerdo completo de un joven de 12 años, bajo el mismo estudio y codificado del mismo modo. 
Figura 7

\section{SCARDAMALIA AND C. BEREITER}

\section{Topic. Should Children Have to learn}

to Speak a Second Language

I think it depends on what country they live in. I mosed to Montreal when I was fiv. and we had a lot of fiench every day. and my dad had to learn french at his office. and he bruugnt hume bunks hume elers night to study the french language, and 1 could ve that he was having trouble, and I haven't had french before until I moved to Munceal. and when liene levesque went into the provincial election the whole prosince started turning bilingual, and on all the packaging there were two languages, and on the ruad signs there wre hoth finglish and french, and about two years "go the English Innguage started disappearing and like it started being roore and mure trench, and we were finding it more and more difficult to cope with it, like ue covijn't understand the parkoging and ic itarted like no mure tinglish on some things, and all Fremch, and then started mustly French lelevision, and we were finding it hard to learn the frunch lankuake. like my dod is old. und he was linding a hard to kearn it and I was lindillg 4 hard. se . decided to move back here uguth, and I think that if you have no other oppurtunity to like. move. alld gou can't ho to a schuol that's English, then you can't do anything ulse hut luarn the f'rench language, or any uther language that's not your owh. then you just have to, you have no other choice.

Parte de un protocolo obtenido en las mismas condiciones que el protocolo del adulto de la figura 6

Este protocolo es el único caso, entre los alumnos de sexto grado, en el que el número de palabras en el protocolo sobrepasa, en más de 10 , a las escritas en el texto. Ninguna actividad adicional es de la clase que encontramos en el protocolo adulto. Excepto los fragmentos que no están marcados, todo el resto es una paráfrasis de los contenidos del texto. Es increíble que los alumnos jóvenes recuerden tanto de sus textos como los adultos. Aparentemente, los escritores jóvenes no se apartan mucho de una cadena de ideas fácilmente accesible para la generación de sus textos, por lo que no tienen que retroceder mucho cuando se trata de recordar el texto. En comparación, los adultos trabajan mucho más, siguiendo las huellas de un complejo laberinto de ideas, y, a pesar de todo ese esfuerzo, no recuerdan más del contenido.

Nótese que, debido a que los escritores jóvenes escribieron composiciones cortas, tienen menos que recordar. Se podría, por lo tanto, argumentar que podrían disponer de las representaciones textuales más abstractas, pero que no tienen necesidad de utilizarlas. Sin embargo, si nuestro análisis global es correcto, los escritores inmaduros también utilizan menos representaciones abstractas durante la generación del texto, lo que implica que sus textos sean más cortos y menos complejos. Por lo tanto, en vez de intentar «desenredar» las diferencias textuales y las del proceso, por medio de manipulaciones experimentales, parecería mejor seguir compro- 
bando otras implicaciones de los dos modelos aquí presentados.

\section{Las representaciones utilizadas}

En el protocolo adulto de la figura 6, el escritor hace referencia a intenciones (ej. «Intentaba poner todas esas cosas juntas»), a claves (señalado, por ejemplo, por: «decir algo así como...») y también a aspectos estructurales del texto (ej. «tercer párrafo»). No obstante, el protocolo del alumno de sexto grado, muestra sólo el recuerdo palabra por palabra o la paráfrasis del material en el texto -lo que llamaremos: representaciones del contenido-. Protocolos de recuerdo de todos los 36 alumnos en esta investigación, fueron evaluados según el grado de intervención del recuerdo, en cada una de las clases de representaciones ya mencionadas: intención, claves, estructura y contenido. Se determinó el mayor número de diferentes clases de representación (máximo 4). Una combinación de los cuatro tipos que representa la interconexión absoluta entre distintas formas de representación, se encontró, con una sola excepción, entre los adultos. Los protocolos de los niños mostraban pocas referencias a claves e intención y la ausencia de la rica cadena de ideas que interviene en los adultos. En la mayoría de los casos, los alumnos de los dos grupos jóvenes, mostraron un acceso directo al contenido de sus textos.

Probablemente, las transformaciones de las ideas son posibles al representar un texto de un modo abstracto y substancial. Tales representaciones liberan al autor de la necesidad de mantener en la memoria activa una representación de los contenidos literales del texto. Si esto es cierto, se deduce que los alumnos jóvenes deben utilizar la capacidad de la memoria a corto plazo simplemente para conservar sus ideas, dejando poca, o ninguna, capacidad excedente, para contrarrestar ideas y para considerar alternativas.

$\mathrm{Al}$ construir representaciones de más alto nivel, los escritores maduros también se proveen de una fuente de recuperación de pistas de la memoria que no se obtiene sólo del propio texto. Flower y Hayes (1981) encontraron que los escritores expertos generan la mayoría de las ideas del contenido respondiendo a sus propias elaboraciones del problema retórico, mientras que los escritores novatos generan el $70 \%$ de sus ideas en respuesta, o al tópico asignado, o al último item del contenido considerado. Eso sugiere, que el modelo «decir el conocimiento» no sólo se limita a un conjunto mucho más pequeño de estimulaciones de la memoria, sino que, también se limita a estimulaciones que son menos probables de relacionarse con objetivos de alto nivel o con puntos de la composición.

\section{Los puntos más importantes en los textos}

De Beaugrande (1984) supone que la representación mental de las ideas principales juega un papel significante en la generación de un texto, es lo que él llama ideas. Estos «puntos principales» son las proposiciones centrales alrededor de las cuales se organiza el texto y que otras proposiciones intentan sustentar. Desde el punto de vista del proceso de composición, lo que interesa no es cómo los puntos principales se presentan en el texto, sino cómo funcionan en la mente del escritor. Se espera de un escritor hábil que los puntos principales funcionen como objetivos, ejerciendo una influencia directiva sobre el proceso de composición. Sin embargo, no es muy seguro que los puntos principales se encuentren representados en la mente de los escritores inmaduros (prescindiendo de si ellos son recuperables de los textos que tales escritores producen). El modelo «decir el conocimiento" podría predecir que los puntos principales no jugaran un papel en los procesos de composición de los escritores inmaduros, siendo los tópicos y los esquemas de género los que se harían cargo del trabajo de mantener la unificación del texto.

Las representaciones mentales de los puntos principales en los escritores, fueron investigadas también, indirectamente, por medio de tareas de recuerdo (Bereiter, Burtis, Scardamalia y Bert, 1983). Los alumnos escribieron un ensayo y, posteriormente, indicaron sus puntos principales. Después, los experimentadores prepararon tres versiones distintas del 
punto principal de cada ensayo. Una versión denorninada «versión aumentada», fue un intento de sonsacar del alumno un comentario del tipo: «Ah, eso es precisamente lo que estaba tratando de decir. Esto aclara el punto principal, mejor de lo que yo lo he hecho». La versión disminuida tenía intención de sonsacar algo así como: «Ese no es mi punto principal. -Quizás haya mencionado que los socorristas consiguen un hermoso bronceado, pero, en cierto modo, sólo lo añadí. No es importante». La tercera versión fue una paráfrasis de la del alumno y esperábamos que la res. puesta fuera del tipo: «Sí, eso es lo que estaba intentando decir».

La suposición fundamental del estudio era la de que si los alumnos construían y utilizaban representaciones de los puntos principales debían ser precisos al reconocer distorsiones de su punto y basar sus juicios en las intenciones que recordaban. Sin embargo, los alumnos que no construyeron o utilizaron tales representaciones, deberían estar más dispuestos a aceptar cualquier versión que fuera compatible con el contenido del texto y no deberían remitirse a las intenciones que recordaban. El estudio fue llevado a cabo con alumnos de quinto y onceavo curso. La mayoría de los alumnos del onceavo curso se comportaron como es de esperar de gente que posee representaciones mentales activas de sus puntos principales. Ellos preferían, con suma confianza, las versiones aumentadas o las que parafraseaban sus declaraciones del punto principal a las versiones disminuidas y podían indicar que ciertas proposiciones, aunque incluidas en sus textos, no eran las que ellos propusieron como puntos principales. Los alumnos de quinto curso, sin embargo, tendieron a aceptar todas las versiones del mismo modo y pocas veces recurrieron a sus propias intenciones al juzgarlas. Era como si se encontraran en la posición de lectores y no de creadores de sus propios textos.

De estos y otros estudios (véase para más datos, Scardamalia y Paris, 1985), surge un cuadro de las representaciones mentales que es consecuente y que añade profundidad psicológica a los cuadros ya descritos. Consecuente con la idea de que «decir el conoci- miento» es un proceso focalizado en generar un texto dato-por-dato, las representaciones mentales de los propios textos de los escritores jóvenes se limitan, mayoritariamente, a datos específicos del contenido o de la expresión lingüística. La única otra clase de representación, que aparece con alguna frecuencia en el recuerdo del texto, es la representación de elementos estructurales (razón, ejemplo, etc.). Esto es consecuente con el papel orientador de la memoria, asignado a tal información, en el modelo «decir el conocimiento». Los escritores maduros muestran el rango completo de representaciones mentales sugeridas en las descripciones generales del proceso de composición (de Beaugrande, 1984). Más aún, sus protocolos de recuerdo muestran que las distintas clases de representaciones están conectadas: los adultos, por ejemplo, recurren a las intenciones o a las claves, en su esfuerzo para reconstruir el texto palabra por palabra. Suponemos que esta interconexión de las representaciones les permite trabajar a distintos niveles - problemas de significado, de expresión, de organizaciónde un modo coordinado y sin perder el dominio del proceso como un todo.

A la luz de estos descubrimientos, sería tentador concluir que la educación de la composición debería centrarse en ayudar a los estudiantes a construir representaciones de sus textos de más alto nivel -especialmente, representaciones de intenciones o de objetivos y de claves o de puntos principales-. Pero, por supuesto que no es el simple hecho de poseer tales representaciones lo que convierte a un escritor en maduro, sino la capacidad de poder operar con ellos. Si los alumnos se encuentran encerrados dentro del proceso «decir el conocimiento», las representaciones de más alto nivel no tienen función alguna. En tal caso, no queda claro cómo pueden los alumnos aprender a crear tales representaciones. Por lo tanto, volvemos a la conclusión de que lo que tiene que desarrollarse es el proceso de composición como un todo, y no algún aspecto o componente de él. Más que señalar un foco particular para educar la composición, la investigación sobre las representaciones mentales contribuye a enten- 
der lo que significa el desarrollo global del proceso de composición hacia niveles más altos.

\section{EL CAMBIO DEL ALUMNO EN SU APROXIMACION A LA ESCRITURA DE «DECIR EL CONOCIMIENTO» HACIA «TRANSFORMAR EL . CONONCIMENTO»}

Si las aproximaciones contrastadas derivan de estructuras cognitivas cualitativamente diferentes y la estructura más avanzada no es una consecuencia directa de la otra, probablemente no bastará con experiencias ricas y variadas de composición, ni con motivaciones para mejorar. $Y$ aunque ellas fueran suficientes para algunos alumnos, habría otros que sacarían más provecho de otro tipo de ayuda.

En nuestra investigación educativa, hemos desarrollado dos intervenciones generales que están detalladas en Bereiter y Scardamali (1987). Una introduce los llamados «ciclos reflexivos» dentro de las rutinas de composición de los niños. La idea es la de modificar el desarrollo secuencial de «decir el conocimiento», haciendo que el alumno re-piense las decisiones, considere alternativas y dirija su atención hacia aspectos de la tarea de composición distintos de la generación del próximo dato de contenido. El ejemplo más claro proviene de un estudio en el que los alumnos tenían que detenerse después de cada frase y seleccionar, de un conjunto de posibilidades, una expresión que cumple funciones evaluativas (Scardamalia y Bereiter, 1983b).

Se espera que tales actividades reflexivas induzcan al alumno a crear pequeños espacios de problemas para tratar los temas que la reflexión descubre, y que de esta manera se generen los espacios de problemas retóricos y de contenido, que constituyan la cualidad estructural principal del modelo «transformar el conocimiento».

La otra intervención para lograr el cambio estructural se basaba en hacer que los alumnos percibieran sus propios procesos de com- posición (Scardamalia y Bereiter, 1983a) y fueran expuestos a los procesos de composición más maduros. Es difícil de esperar que los niños, excepto aquellos afortunados que están en contacto con escritores profesionales, perciban las complejidades subyacentes al quehacer de los expertos. Nuestro objetivo primordial al idear la instrucción, fue que conocieran este mundo. (Una exposición más completa de los procedimientos de la investigación y de sus resultados se encuentra en Scardamalia et al., 1984.)

\section{La exposición a procesos de composición maduros}

Los alumnos sugirieron tópicos para los ensayos - por ejemplo, «Las estrellas de Rock son hoy día más talentosos que nunca»Después observaron a un adulto, pensando en voz alta, mientras se esforzaba en escribir un ensayo sobre este tópico. El adulto podría decir lo siguiente:

No sé absolutamente nada sobre estrellas modernas de Rock. No me puedo acordar ni siquiera de un solo nombre de una estrella de Rock. Qué de David Bowie o Mick Jagger... Pero muchos lectores no estarían de acuerdo en que... sean estrella de Rock modernas. Creo que ambos son tan mayores como lo soy yo. Veremos, mis sentimientos personales sobre esto son... que dudo mucho de si las estrellas de Rock de hoy día son más talentosas que nunca. De todos modos, cómo podría saberlo. Podría argumentar que... Necesito una idea nueva... Un punto importante que no he tenido en cuenta todavía es... ah.. bien... ¿qué queremos decir por talento? ¿Estoy hablando de talento musical o de la habilidad de entretener - de hacer acrobática? Ey, quizás tenga un camino para este tópico. Podría desarrollar esta idea por medio...

El modelado propiamente dicho era mucho más complicado. Este fragmento corto, llevó el tiempo real de 10 minutos.

\section{El andamiaje de los procesos maduros}

Para ayudar a los alumnos a planificar y a que vayan más allá del «¿qué sigue?» en la generación del contenido, les proporcionamos tarjetas que contenían sugerencias que podían 
incorporar a sus pensamientos. Las frases de las sugerencias para la planificación de los enlos educadores en sus monólogos de planifi- sayos de opinión y, en la figura 9, una muescación, fueron algunas de las sugerencias uti- tra de un conjunto paralelo utilizado para exlizadas. En la figura 8 vemos una muestra de poner los hechos.

FiguRA 8

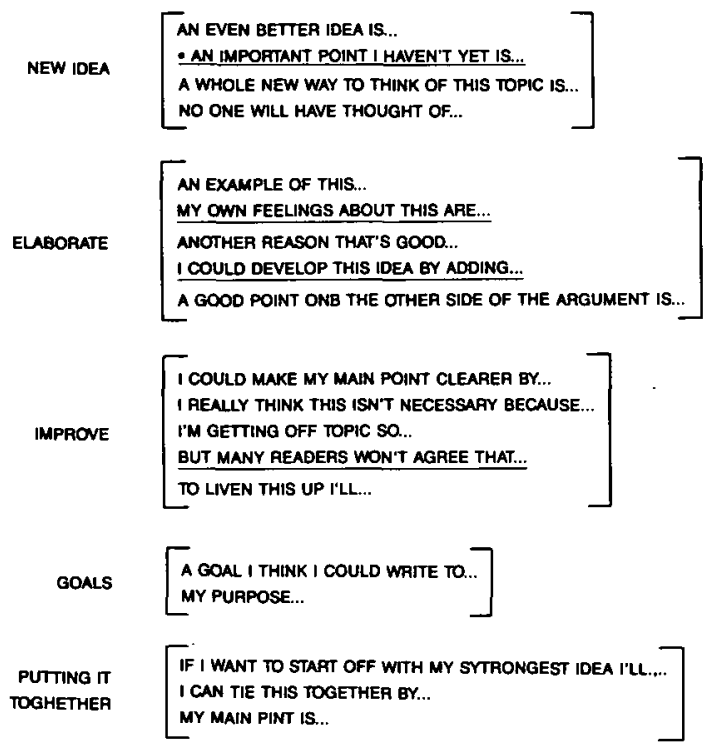

Ejemplos de sugerencias para ayudar a los estudiantes a planificar sus ensayos

FigURA 9

\begin{tabular}{|c|c|}
\hline NEW IDEA & $\begin{array}{l}\text { AN IMPORTANT DISTINCTION IS... } \\
\text { A CONSEOUENCE OF (THIS IS)... } \\
\text { THE HISTORY OF THIS IS... } \\
\text { SOMETHING THAT IS SIMILAR IS... } \\
\text { A CAUSE OF (THIS IS)... }\end{array}$ \\
\hline LABORATE & $\begin{array}{l}\text { I'M IMPRESSED BY... } \\
\text { AN EXPLANATION WOULD BE... } \\
\text { AN EXAMPLE OF (THIS IS)... } \\
\text { THIS RESULTS IN... } \\
\text { MY OWN EXPERIENCE WITH THIS IS... }\end{array}$ \\
\hline IMPROVE & $\begin{array}{l}\text { I COULD DESCRIBE THIS IN MORE DETAIL GY ADDING... } \\
\text { THIS ISN'T EXACTLY HOW IT IS BECAUSE... } \\
\text { I COULD GIVE THE READER A CLEAR PICTURE BY... } \\
\text { THIS ISN'T TRUE OF ALL... } \\
\text { TO PUT IT MORE SIMPLY... }\end{array}$ \\
\hline GOALS & $\begin{array}{l}\text { MGOAL I THINK I COULO WAITE TO... } \\
\text { MY PURPOSE... }\end{array}$ \\
\hline $\begin{array}{l}\text { PUTTING IT } \\
\text { TOGETHER }\end{array}$ & $\begin{array}{l}\text { IF I WANT TO STAAT OFF WITH MY STRONGEST IDEA I'LL... } \\
\text { I CAN TIE THIS TOGETHER GY... } \\
\text { MY MAIN POINT IS... }\end{array}$ \\
\hline
\end{tabular}


Cada alumno tenía en las tarjetas índice, un conjunto amplio de frases pertenecientes al género particular con el que se trabaja. Todo modelado -incluido aquel hecho por los adultos - se llevó a cabo utilizando las tarjetas. Esta planificación proveía alumnos ayudantes que, por turnos voluntarios, pensaban en voz alta en frente de la clase. La actividad se convirtió en la preferida y los alumnos esperaban ansiosos su turno, en lo que se llegó a llamar «monologando».

\section{Distintas actividades mentales de control que necesitan atención}

Al principio, los alumnos elegían las tarjetas al azar. Al tener, con la ayuda de las tarjetas, más práctica en la planificación, fueron animados a controlar sus propios pensamientos, para determinar si necesitaban una nueva idea, o si necesitaban elaborar o improvisar una idea, o si necesitaban evaluar sus objetivos, o intentar unir todo lo que ya tenían. Las tarjetas se subdividieron en las categorías indicadas en las figuras 10 y 11, para ayudar a este proceso. Los alumnos aprendieron a seleccionar las tarjetas de acuerdo con la clase de idea que necesitaban.

\section{La interacción entre el espacio de contenido y el retórico}

Entre las sugerencias para la planificación, fueron incorporados algunos intereses retóricos, incluyendo las obligaciones que surgían de las expectativas del género, de los del lector y de los de sentimientos personales. Frases tales como «nadie hubiera pensado en» y «Una diferencia importante es», llevaban a los alumnos, la mayoría de las veces, a recuperar una información que les sorprendía o que les deleitaba. Parecía, por lo tanto, que tales sugerencias estaban consiguiendo la intención de poner en marcha los pocesos de transformar el conocimiento que resultan de la interacción entre incumbencias retóricas y de contenido. $\mathrm{O}$ sea que, las incumbencias retóricas estimuladas por las sugerencias que había en las tarjetas, estaban siendo trasladadas a estimulaciones del contenido, que hacían surgir conexiones no reconocidas ante- riormente. El educador intentaba, por medio del andamiaje y de la explicación, alentar ciclos repetitivos y de repensamiento, de la clase que se encuentra en los protocolos de los escritores expertos. También se animaba a los alumnos a actuar de la forma más independiente posible. Las sugerencias para la planificación se veían como recursos transitorios para este propósito. Proveían un medio de apoyo que permitía a los alumnos planificar sus composiciones sin ninguna guía del maestro. Como era de esperar, los alumnos dependían cada vez menos de las sugerencias en las tarjetas, planificando solos durante largos períodos de tiempo, y recurriendo a las tarjetas sólo cuando estaban «atascados».

Este experimento produjo diferencias significativas tanto en los protocolos registrados en voz alta como en los ensayos escritos. El grupo experimental mostró una mayor ventaja, al otorgársele un tiempo ilimitado para la planificación, moviéndose desde las características inmediatas del comienzo de decir el conocimiento hasta el punto donde pasaban, espontáneamente, hasta cuatro períodos de clases planificando antes de comenzar a redactar un ensayo mayor. Incluso en situaciones de tiempo limitado, sus ensayos expositivos mostraron un nivel de reflexión significativamente mayor a la que mostraban los de los controles, aunque esta reflexión no se encontrara en sus ensayos de opinión. En general, la poca consistencia en los ensayos de los alumnos del grupo experimental, podría derivar de las dificultades para mantener la coherencia. Esto lo atribuimos, al esfuerzo de recuperar una información cada vez más variada, algo que no estaban acostumbrados a controlar.

\section{CONCLUSIONES}

Los escritores novatos dependen para sus composiciones de que el conocimiento se encuentre ya agrupado (ya sea en la memoria o por medio de actividades dirigidas por los maestros) en formas preparadas para la presentación escrita. Los experimentados pueden hacer uso de procedimientos complejos de elaboración del conocimiento para transfor- 
marlo aunque no esté agrupado de forma coherente y efectiva. Por lo tanto, lo que vemos en la obra de los escritores experimentados, es la ejecución de procedimientos poderosos que les permiten delimitar, elaborar y refinar conocimientos disponibles. Para los novatos, sin embargo, la composición sirve, más bien, para reproducir que para refinar el conocimiento.

En este artículo hemos presentado dos modelos distintivos de estas aproximaciones y revisado los datos de confección de borradores, de planificación y de revisión, que sutentan estos dos modelos. También descubrimos evidencias de las grandes diferencias entre las representaciones mentales de un texto que fundamentan estos modelos. Finalmente, presentamos resultados de investigaciones educativas, cuya finalidad fue la de fomentar una aproximación que transforme el conocimiento.
Esta investigación sugiere que es necesario un orden adicional de objetivos educativos. Si queremos ayudar a los alumnos a adquirir una estructura de «transformar el conocimiento» para la composición, necesitan modelos explícitos de competencia madura y medios para habérselas con procedimientos que amplían la competencia que ellos traen a la tarea espontánea. Los esfuerzos educativos sugieren que es posible transmitir los procedimientos complejos de solución de problemas que poseen los expertos, y que al hacerlo se pueden ampliar las facultades de los alumnos para escribir. Sin embargo, los estudios educativos también sugieren que pasar de «decir el conocimiento» a «transformar el conocimiento» en la composición, no es un proceso de crecimiento sino que es, más bien, la reconstrucción de una estructura cognitiva.

\section{Notas}

1 Una versión anterior de este artículo fue publicada en 1987 en S. Rosenberg (Ed.). Advances in applied psycholonguistics. Vol. 2. Reading, writing and language learning (pp. 142-1.750). Cambridge: Cambridge University Press.

\section{Referencias}

Anderson. J. R. (1983). The architecture of cognition. Cambridge, MA: Harvard University Press.

Bereiter. C; Burtis, P. J; Scardamalia, M., y Brett. C. (1983, abril). Developmental differences in mental representation of main point in composition. Documento presentado en el congreso de American Educational Research Association, Montreal.

Bereiter, C., y SCardamalia, M. (1987). The psychology of written composition. Hillsdale, N. J.: Erlbaum.

BritTon, J. (1982). Spectator role and the beginnings of writins. En M. Nystrand (Ed.), What writers know: The language, process, and structure of written discourse (pp. 149-69). Nueva York. Academic Press.

Bryant, D. C. (1965). Rhetoric: Its function and scope. En J. Schwartz y J. A. Rycenga (Eds.), The province of rhetoric (pp. 3-36). Ronald Press. Nueva York.

BurTis, P. J. (1983, abril). Planning in narrative and argument writing. Documento presentado en el congreso de American Educational Research Association, Montreal.

Burtis, P. J; Bereiter, C; Scardamalia, M., y Tetroe, J. (1983). The development of planing in writing. En G. Wells y B. M. Kroll (Eds.), Explorations in the development of writing (pp. 153-74). Wiley. Nueva York.

Caccamise, D. J. (en prensa). Idea generation in writing. En A. Matsuhashi (Ed.), Writing in real time: Modelling production processes. Nueva York. Longman.

Calkins, L. M. (1979). Andrea learns to make writing hard. Language Arts, 56, 569.76.

Calkins, L. M. (1983). Lessons from a child: On the teaching and learning of writing. Exeter, N. H.: Heinemann.

Chомsкy, N. (1980). Rules and representations. Nueva York.Columbia University Press.

Collins, A. N., y Loftus, E. F. (1975). A spreading activation theory of semantic processing. Psychological Review, $82,407.28$.

De Beaugrande, R. (1984). Text production: Toward a science of composition. Norwood, N. J.: Ablex.

Elbow. P. (1973). Writing without teachers. Nueva York. Oxford University Press.

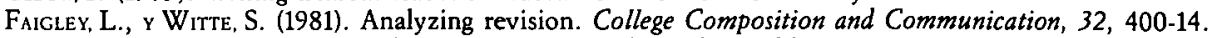

FLOWER, L. S. (1979). Writer-based prose: A cognitive basis for problems in writing. College English, 41, 19-37.

Flower, L. S., y Hayes. J. R. (1980). The cognition of discovery: Defining a rhetorical problem. College Composition and Communication, 31, 21.32.

Flower. L. S., Y HaYes, J. R. (1981). The pregnant pause: An inquiry into the nature of planning. Research in the Teaching of English, 15, 229.44.

Flower, L. S., y HAYEs, J. R. (1984). Images, plans and prose: The representation of meaning in writing. Written Communication, $1,120 \cdot 60$. 
Graves, D. H. (1979). What children show us about revision. Language Arts, 56, 312-19.

HaYes, J. R., y Flower. L. S. (1980b). Writing as problem solving. Visible Language, 14, 388-99.

Lowenthal, D. (1980). Mixing levels of revision. Visible Language, 14, 383-7.

McCutchen, D., y Perfetri, C. A. (1982). Coherence and connectedness in the development of discourse production. Text, 2, 113-39.

Murray, D. M. (1978). Internal revision: A process of discovery. En C. R. Cooper y L. Odell (Eds.), Research on composing (pp. 85-103). Urbana, IL: National Council of Teachers of English.

National Assessment Of Educational Progress (1977). Write/rewrite: An assessment of revision skills; selected results from the second national assessment of writins. ('Tech. Rep.). Washington, DC: U. S. Government Printing Office. (ERIC Document Reproduction Service No. ED 141 826).

National Assessment Of Educational Progress (1980a). Writing acbievement, 1969-79: Results from the third national writing assessment: Vol. 1. 17-year-olds (Tech. Rep.). Denver, CO: National Assessment of Educational Progress. (ERIC Document Reproduction Service No. ED 196 042).

National Assessment Of Educational Progress (1980b). Writing acbievement, 1969-79: Resutls from the third national writing assessment: Vol. 2. 13-year-olds (Tech. Rep.). Denver, CO: National Assessment of Educational Progress. (ERIC Document Reproduction Service No. ED 196 043).

Newell, A. (1980). Reasoning, problem solving, and decision processes: The problem space as a fundamental category. En R. S. Nickerson (Ed.), Attention and performance VIII (pp. 693-718). Hillsdale, N. J.: Erlbaum.

Nold, E. W. (1981). Revising. En C. H. Frederiksen y J. F. Dominic (Eds.), Writing: The nature, development and teaching of written communication (pp. 67-79). Hillsdale, N. J.: Erlbaum.

Odell, L. (1980). Business writing: Observations and implications for teaching composition. Theory into Practice, $19,225-32$.

PERL, S. (1979). The composing processes of unskilled college writers. Research in the Teaching of English, 13, 317-36.

Scardamalia, M., y Bereiter, C. (1982). Assimilative processes in composition planning. Educational Psychologist, $17,165 \cdot 71$.

Sacardamalia, M., y Bereiter, C. (1983a). Child as co-investigator: Helping children gain insight into their own mental processes. En S. Paris, G. Olson y H. Stevenson (Eds.), Leaming and motivation in the classroom (61-82). Hillsdale, N. J.: Erlbaum.

Scardamalia, M; Bereiter. C., y Steinbach, R. (1984). Teachability of reflective processes in written composition. Cognitive Science, 8, 173-90.

Scardamalia, M., y Paris, P. (1985). The function of explicit discourse knowledge in the development of text representations and composing strategies. Cognition and Instruction, 2, 1.39.

Sommers, N. (1980). Revision strategies of student writers and experienced adult writers. College Composition and Communications, $m$ 31, 378-88.

W RITERS AT WorK: The Paris review interviews (2nd series). (1963). Nueva York: Viking.

ZBRODOFF, N. J. (1984). Writing stories under time and legth constraints. Unpublished doctoral dissertation, University of Toronto.

\section{Extended Summary}

Two models of composing are set forth, one intended to capture essential features of inmature composing and the other features that distinguish mature writers. The contrasting models are called knowledge telling and knowledge transforming. The basic idea underlying the models is that the principal difference between mature and inmature composing is in how knowledge is brought into the writing process and in what happens to knowledge in that process. The psychological plausibility of the two models is addressed by the analysis of subjective accounts of the composing process, and by an account of the way the cognitive structurs described by the models. These might come to be acquired. The characteristics of the resulting texts, the differences in start-up times and the processes of notemaking, planning and revising are interpreted in light of the contrasting models. Two kinds of evidences are discussed for the kind of mental representations underlyng the two models are presented as well as examples of instructional interventions. I's Educational interventions are presented because it is assumed that the applied cognitive scientist task is not merely to describe cognitive processes but to describe them in ways that capture educationally significant differences. The described instructional efforts are based on the premise that helping students to move from a knowledge telling to a knowledge transforming approach to writing is an important and realistic educational objective. It will be argued that the importance of this objective extends beyond writing instruction, having broad implications for the way students develop their knowledge. 